\title{
Effects of Neutralization, Decoloration, and Deodorization on Polycyclic Aromatic Hydrocarbons during Laboratory-Scale Oil Refining Process
}

\author{
Yuxiang Ma, Longkai Shi, Yulan Liu, and Qiyu Lu \\ College of Food Science and Technology, Henan University of Technology, Zhengzhou 450001, China \\ Correspondence should be addressed to Qiyu Lu; qiyulu7120@vip.sina.com
}

Received 16 November 2016; Revised 13 February 2017; Accepted 9 April 2017; Published 28 May 2017

Academic Editor: Feng Xu

Copyright (c) 2017 Yuxiang Ma et al. This is an open access article distributed under the Creative Commons Attribution License, which permits unrestricted use, distribution, and reproduction in any medium, provided the original work is properly cited.

\begin{abstract}
The influence of technological operations during oil refining process on polycyclic aromatic hydrocarbons (PAHs) in neutralized, bleached, and deodorized oils was investigated on the basis of laboratory-scale study. Under the best experimental conditions, benzo[a]pyrene decreased by $85.1 \%, 99.7 \%$, and $40.8 \%$ in neutralized, bleached, and deodorized oils, respectively. Total of 16 analytes decreased by $55.7 \%, 87.5 \%$, and $47.7 \%$, respectively. Bleaching with activated charcoal was the most efficient procedure to reduce PAHs in crude oil. Neutralization had a modest influence on sixteen analytes; however, deodorization was only responsible for a slight decrease in the light PAHs and heavy PAHs contents. Data obtained in this study suggest that the use of activated carbon during oil refining process is highly recommended; moreover, these results provide a useful guidance for oil refining plant to reduce security risk and ensure the quality of the vegetable oil products.
\end{abstract}

\section{Introduction}

Edible vegetable oils consist of a complex mixture of glycerides (mainly triacylglycerols and partially diacylglycerols and monoacylglycerols), hydrocarbons, alcohols, phospholipids, free fatty acids, pigments, sterols, tocopherols, and volatile compounds, and so on $[1,2]$. Usually, oil refining process is done to eliminate the unwanted minor components, which make oils not suitable for sale and consumption. Furthermore, the purification process is trying to reduce possible damage to the neutral oil as well as minimize refining loss $[3,4]$. All compounds, which are detrimental to flavour, color, stability, and safety of the refined final oil products will be removed. They are primarily phospholipids, free fatty acids, pigments, trace metals, volatiles, persistent organic pollutants (POPs), and other impurities $[1,5]$.

Among various POPs, polycyclic aromatic hydrocarbons (PAHs) are a class of ubiquitous chemical contaminations in vegetable oils. Academically, they are divided into two parts, light PAHs (LPAHs, up to four fused rings) and heavy PAHs (HPAHs, more than four fused rings). The persistent nature of PAHs related to their great chemical stability leads to their significant accumulation in the food chain; moreover, their high liposolubility results in their occurrence in oils and fatty foods, particularly in edible oils. In 2005, China has set a limit of $10 \mu \mathrm{g} / \mathrm{kg}$ for benzo[a]pyrene (BaP) in edible vegetable oils for the sake of dietary safety associated with oil consumption [6]. Meanwhile, Commission Regulation Number 835/2011 legislated the maximum levels for PAHs in fats and oils for direct human consumption or ingredient for food products [7]. It established $2 \mu \mathrm{g} / \mathrm{kg}$ for $\mathrm{BaP}$ and $10 \mu \mathrm{g} / \mathrm{kg}$ for summed four PAHs (called $\mathrm{PAH}_{4}$, including $\mathrm{BaP}$, benzo[b]fluoranthene (BbFlu), benz[a]anthracene $(\mathrm{BaA})$, and chrysene $(\mathrm{Chr})$ ) as the most suitable indicator of PAHs in foodstuffs. However, no countries or organizations worldwide have set the regulations and limits for the content of total PAHs in commercial oils, partly due to the fact that there are hundreds of these sorts of pollutants in the world, thus resulting in difficulty in separation, determination, and calculation. Previously, we developed and validated a liquid chromatography-atmospheric pressure photoionizationtandem mass spectrometry method for analyzing United 
States Environmental Protection Agency (EPA) 16 PAHs in edible oils [8], and the occurrence as well as concentration of 16 analytes in eighty-five brands of vegetable oils in China was detected and statistically analyzed [9]. Now, investigations on technologies for the removal of PAHs from edible oils reach considerable importance for reducing health risks to consumers and the efficiency related to PAHs reduction is still questioned due to the fact that available information regarding specific process conditions is limited.

Normally, the amount of PAHs in crude vegetable oils can be eliminated during oil refining process, thus generating low levels of contamination in the final oil products compared to in the crude ones [10]. In China, conventional oil refining process consists of four steps (i.e., degumming, neutralization, decoloration, and deodorization), and the last three procedures were reported with good removing effect on PAHs in edible vegetable oils [10-14]. Neutralization, also known as alkali refining, is used to eliminate free fatty acids that can promote lipid oxidation by forming soapstock. Adsorbents, such as activated clay and activated carbon, can be used to remove oil-solubility pigments (e.g., carotene and chlorophyll), metals, and oxidation products as well as remaining phospholipids and soaps, and this procedure is defined as decoloration or bleaching. Deodorization, or sometimes called steam distillation, removes volatile components and small molecular decomposed peroxides at elevated temperature under vacuum to improve flavour quality, shelf life, and stability of the final oil products.

The aim of this study is to investigate the effects of three main steps, namely, neutralization, decoloration, and deodorization, on PAHs in crude vegetable oils, to examine the respective influence of parameters employed on their efficiencies in reducing of PAHs, and to characterize the distribution and evaluate the behaviour of PAHs during the whole technological procedure adopted. This is a comprehensive effort to report on the levels of selected PAHs measured in different stages of the oil refining. Meanwhile, the inclusion of multiple PAHs provides a full-scale representation of the entire group of pollutants in edible oils during the refining process.

\section{Materials and Methods}

2.1. Materials and Chemicals. Mixture of EPA 16 PAHs was provided by O2si (Charleston, South Carolina, USA) with the concentration of $200 \mu \mathrm{g} / \mathrm{mL}$ (dissolved in acetonitrile); the full names and abbreviations of 16 analytes related to this study are shown in Table 1. Sixteen corresponding isotopical analogues were obtained from Dr. Ehrenstorfer $\mathrm{GmbH}$ (Augsburg, Germany) and C/D/N Isotopes (PointeClaire, Quebec, Canada). Purities of individual standard components were guaranteed to be above $97.9 \%$.

Acetonitrile and toluene (HPLC grade) were purchased from Sigma (St. Louis, MO, USA). Deionized water was obtained from a Millipore Milli-Q water purification system (Billerica, MA, USA). Degummed peanut oil sample with a high contamination level of PAHs, which was used for neutralization, bleaching, and deodorization, was prepared
TABLE 1: The full names and abbreviations of EPA 16 PAHs related to this study.

\begin{tabular}{lc}
\hline PAH & Abbreviation \\
\hline Naphthalene & Nap \\
Acenaphthylene & Acy \\
Acenaphthene & Ace \\
Fluorene & Fl \\
Phenanthrene & Phe \\
Anthracene & Ant \\
Fluoranthene & Flu \\
Pyrene & Pyr \\
Benz[a]anthracene & $\mathrm{BaA}$ \\
Chrysene & $\mathrm{Chr}$ \\
Benzo[b]fluoranthene & $\mathrm{BbFlu}$ \\
Benzo[k]fluoranthene & $\mathrm{BkFlu}$ \\
Benzo[a]pyrene & $\mathrm{BaP}$ \\
Dibenz[a,h]anthracene & $\mathrm{DBahA}$ \\
Benzo[ghi]perylene & $\mathrm{BghiP}$ \\
Indeno[1,2,3-cd]pyrene & $\mathrm{IP}$ \\
\hline
\end{tabular}

in our own laboratory. Potassium hydroxide $(\mathrm{KOH})$ used for alkali refining was purchased from Merck Chemicals Co., Ltd. (Shanghai, China). In this study, three absorbents were chosen as they represent some of the types used by refineries in China. Activated clay (AC) was purchased from Maige Adsorbents Co., Ltd. (Jiangsu, China). Notit-8015 activated charcoal (NACc) was obtained from Zhongji Chemicals Import \& Export Co., Ltd. (Shanghai, China). WY activated charcoal (WYACc) was obtained from Chongqing Beyond Ocean Carbon Manufacture Co., Ltd. (Chongqing, China).

2.2. Oil Refining Process. Fifty grams of prepared oils was used for each experiment. Three oil refining procedures were respectively operated with the prepared degummed oil sample; thus the efficiency of individual steps for removing PAHs could be obtained and compared. A Flowchart for experimental design and all parameters is shown in Figure 1. In this study, orthogonal experiments were employed to select the best removal conditions for the two former processes (neutralization and decoloration). Nine experiments were performed for each alkali refining and bleaching based on the standard $\mathrm{L}_{9}$ orthogonal arrays, whereas single factor experiment was used to optimize the counterpart for deodorization.

2.2.1. Neutralization. The indicated concentration of $\mathrm{KOH}$ was add to the prepared oil sample to neutralize the free fatty acids and the desired concentration of additional amount of $\mathrm{KOH}$ was infused to ensure the complete formation of soaps. This was proceeded with agitation at $40^{\circ} \mathrm{C}$ originally, and once the $\mathrm{KOH}$ was added, the temperature was increased to the indicated temperature immediately, maintaining the temperature for the desired time. Then the soaps were separated by centrifugation at $5400 \mathrm{r} / \mathrm{min}$. To remove the traces of soaps dissolved in the treated oil and the remains of the basic reagents added, the oils obtained in the previous 


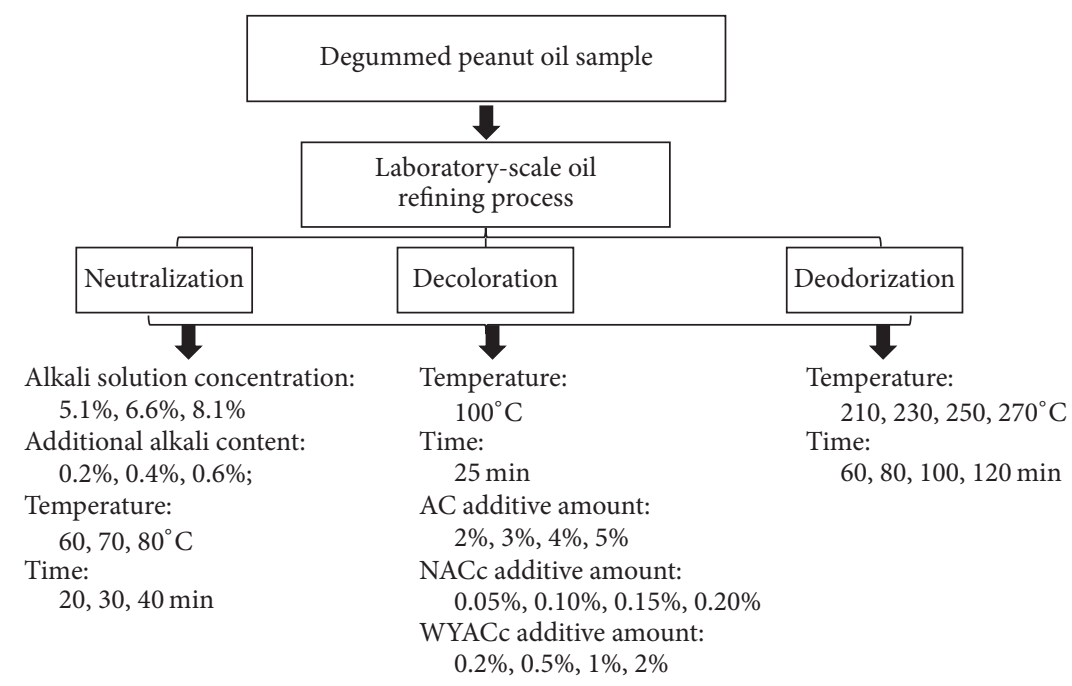

FIGURE 1: Flowchart for experimental design and all parameters.

stages were washed with three successive additions of $10 \%$ of $95^{\circ} \mathrm{C}$ pure water. Then removing the washing water by centrifuging and the neutralized oil was obtained.

2.2.2. Decoloration. The removal of PAHs by the oil bleaching procedure was compared using three commercial sorbents: AC, NACc, and WYACc. According to the actual amount of these three kinds of processing agents used in the oil refinery, the proportions of AC, NACc, and WYACc were optimized and set between 2 and 4\%, 0.05 and $0.2 \%$, and 0.2 and $2 \%$, respectively. Vacuum bleaching was performed in 30-35 mbar by using a vacuum pump and the treatment process was as follows: the adopted adsorbent was add to the prepared oil sample, and the mixture was maintained by agitation for the desired time at the indicated temperature in a vacuum atmosphere. Then the adsorbents were separated by centrifugation at $5400 \mathrm{r} / \mathrm{min}$, and the bleached oil was obtained.

2.2.3. Deodorization. The deodorization was accomplished at the indicated temperature and $80 \mathrm{~Pa}$ with the use of direct stream $(20 \mathrm{~kg} / \mathrm{T} \cdot \mathrm{h})$ in order to remove volatile compounds and thermolabile pigments. Under the high temperature and high vacuum conditions, tested oil was maintained for the desired time and then cooled down to ambient temperature and the deodorized oil was obtained.

\subsection{Oil PAHs Determination and Analytical Conditions. Oil} PAHs determination scheme and analytical means were described in detail in a previous published paper [8]; all of the oil samples were prepared and determined in triplicate. A flowchart describing the sample preparation and purification procedure is provided in Figure 2. Ion pairs of 16 analytes and their analogous are given in Table S1 (Supplementary Material, available online at https://doi.org/10.1155/2017/7824761); data of the method validation are provided in Tables S2 and S3. Briefly, the calibration curves of each analyte showed a linear response with correlation coefficients between 0.9992 and 0.9999 . The recoveries of 16 analytes at three spiking levels were ranging from 77.8 to $106.4 \%$, and the relative standard deviations were lower than $10 \%$. The limits of quantitation for 16 PAHs were in the range of $0.02-0.43 \mu \mathrm{g} / \mathrm{kg}$. Table S4 shows the LC conditions and all of the required instrumental parameters. Data analysis was performed using Origin 8.0 and Microsoft Office Excel 2015.

\section{Results and Discussion}

3.1. Effects of Neutralization on PAHs. As it is known, parameters (alkali concentration, amount of additional alkali, temperature, and time) of the alkali refining process have a significant impact on the quantity and composition of the neutralized oil. An $\mathrm{L}_{9}\left(3^{4}\right)$ orthogonal matrix with four factors, each factor containing three levels, was selected to arrange the experiments. Alkali solution concentrations were $5.1 \%, 6.6 \%$, and $8.1 \%$; additional alkali contents were $0.2 \%$, $0.4 \%$, and $0.6 \%$; temperatures were 60,70 , and $80^{\circ} \mathrm{C}$; times were 20,30 , and $40 \mathrm{~min}$. The levels for each parameter variables were selected from a series of preliminary trials without using particular experimental designs (data were not shown).

Results of experiments in various conditions are displayed in Table 2. Optimum condition of each parameter was shown as the highest value of the removal rate. The optimum conditions for elimination of $\mathrm{BaP}, \mathrm{LPAHs}$, and $\mathrm{PAH}_{16}$ were achieved at alkali concentration of $5.1 \%$, additional alkali amount of $0.6 \%$, temperature of $80^{\circ} \mathrm{C}$, and time of $40 \mathrm{~min}$. For HPAHs, the best results were achieved at alkali concentration of $6.6 \%$, additional alkali amount of $0.6 \%$, temperature of $70^{\circ} \mathrm{C}$, and time of $20 \mathrm{~min}$. And for $\mathrm{PAH}_{4}$, the best conditions were alkali concentration of $5.1 \%$, additional alkali amount of $0.4 \%$, temperature of $70^{\circ} \mathrm{C}$, and time of $30 \mathrm{~min}$. In addition to the main effect, the signal-to-noise $(\mathrm{S} / \mathrm{N})$ ratio analysis was used to evaluate the quality of the results obtained. S/N 


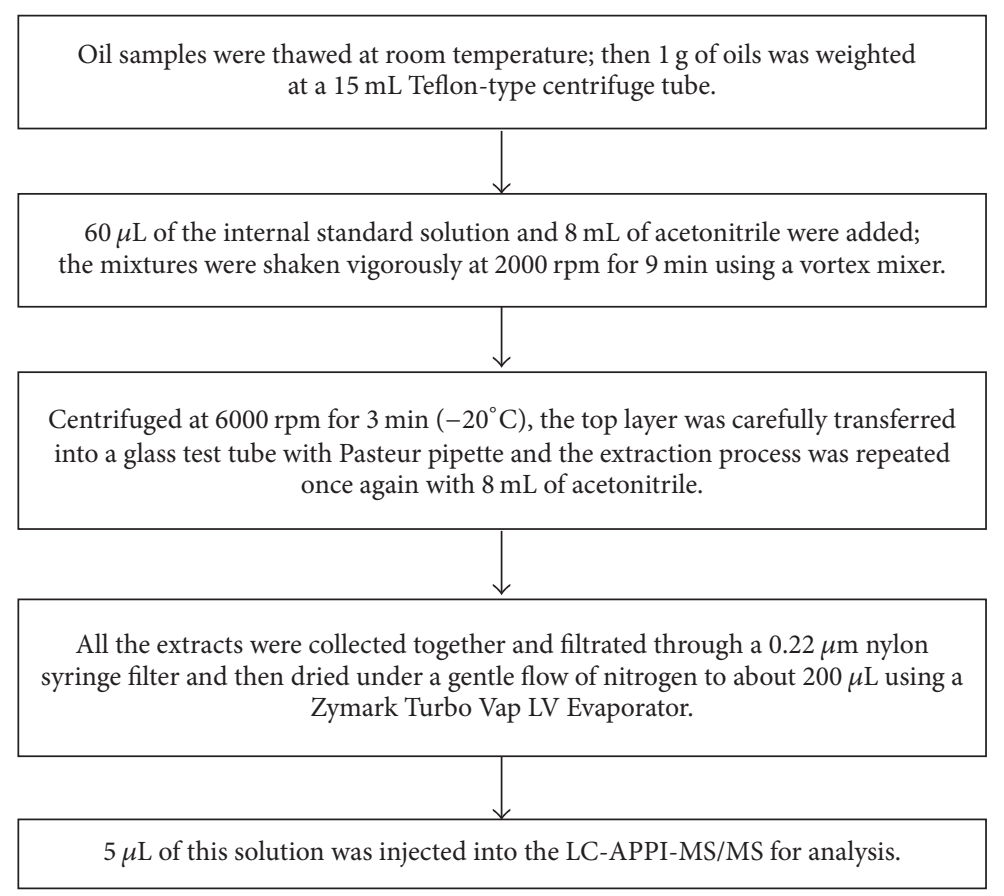

FIGURE 2: Flowchart for oil sample preparation and purification procedure.

ratio with "higher is better" goal was selected to optimize the process. Table 2 also shows the result of $\mathrm{S} / \mathrm{N}$ ratio analysis, and $\mathrm{S} / \mathrm{N}$ ratio analysis revealed that the optimum conditions for the five indicators were not similar to the optimum conditions obtained using the main effect plot. $\mathrm{S} / \mathrm{N}$ ratio analysis showed that time is the highest influence parameter on $\mathrm{BaP}$ and HPAHs, whereas alkali solution concentration is the highest influence parameter on $\mathrm{PAH}_{4}$, LPAHs, and $\mathrm{PAH}_{16}$. Taking everything into consideration, the optimized conditions obtained were alkali concentration of $5.1 \%$, additional alkali amount of $0.2 \%$, temperature of $70^{\circ} \mathrm{C}$, and time of $40 \mathrm{~min}$.

3.2. Effects of Decoloration on PAHs. The effects of three absorbents on PAHs were compared in this study. The temperature and time for single factor experiments were set at $100^{\circ} \mathrm{C}$ and $25 \mathrm{~min}$, respectively. AC additive amounts were $2 \%, 3 \%, 4 \%$, and $5 \%$; NACc additive amounts were $0.05 \%, 0.10 \%, 0.15 \%$, and $0.20 \%$; WYACc additive amounts were $0.2 \%, 0.5 \%, 1 \%$, and $2 \%$. It was observed that increase in added contents of absorbent had a favourable effect on removing concentration of PAHs in crude oils. The removal rates of all three absorbents increase with amount rise at constant time and constant temperature. NACc was the best sorbent (additive amount was $0.2 \%$ ) with the removal rates of 99.1, 78.9\%, 99.1\%, 98.5\%, and 81.5 for BaP, LPAHs, HPAHs, $\mathrm{PAH}_{4}$, and $\mathrm{PAH}_{16}$, respectively. These could be explained by the NACc characteristics and the molecular conformation, namely, the large surface area of NACc that provides suitable pore volume and pore size for adequate adsorption. The data we observed demonstrated that almost all the 4-6 rings analytes can be eliminated by using $0.2 \%$ of NACc. Moreover, the removal efficiencies for analytes less than 4 rings were quite satisfactory (approximately $80 \%$ for individual object), thus proving its high ability to adsorb PAHs in edible oils. However, AC was the weakest one, even if at the biggest quantity (5\%); the removal rates of $\mathrm{BaP}$, LPAHs, HPAHs, $\mathrm{PAH}_{4}$, and $\mathrm{PAH}_{16}$ were 79.7\%, 43.9\%, 83.5\%, 81.6\%, and $48.9 \%$, respectively. Especially in the LPAHs and $\mathrm{PAH}_{16}$, low removal efficiencies (less than 50\%) were observed. In terms of WYACc, comparable results were obtained when $1 \%$ of WYACc was added (compared with $0.2 \%$ of NACc). Individually, the removal rates of BaP, LPAHs, $\mathrm{HPAHs}, \mathrm{PAH}_{4}$, and $\mathrm{PAH}_{16}$ were $99.84 \%, 74.61 \%, 99.38 \%, 98.85 \%$, and $77.74 \%$ when $1 \%$ of WYACc was employed. It is worth mentioning that when $2 \%$ of WYACc was used, almost all the 16 PAHs can be reduced, even the 2-3-ring PAHs. The removal rates of LPAHs and $\mathrm{PAH}_{16}$ were $93.1 \%$ and $94.0 \%$ for $2 \%$ of WYACc.

NACc was the best choice in this study, due to its high efficiency at extremely low content of addition. However, the expensive price of this material is one of the limits to its application in oil bleaching process. For this reason, WYACc, the one possessing the comparative PAHs removal efficiency, was selected to proceed with the orthogonal experiment.

In orthogonal test, parameters including WYACc amount, temperature, and time were optimized. An $\mathrm{L}_{9}\left(3^{4}\right)$ orthogonal matrix with three factors, each factor containing three levels, was selected to arrange the experiments. WYACc amounts were $0.2 \%, 0.5 \%$, and $1.0 \%$; temperatures were 100 , 110 , and $120^{\circ} \mathrm{C}$; times were 15,25 , and $35 \mathrm{~min}$. Results of experiments in various conditions are shown in Table 3. The optimum conditions for elimination of BaP, HPAHs, LPAHs, and $\mathrm{PAH}_{16}$ were achieved at WYACc amount of $1.0 \%$, temperature of $100^{\circ} \mathrm{C}$, and time of $35 \mathrm{~min}$. For $\mathrm{PAH}_{4}$, the best conditions were achieved at WYACc amount of 
TABLE 2: Results of neutralization on PAHs.

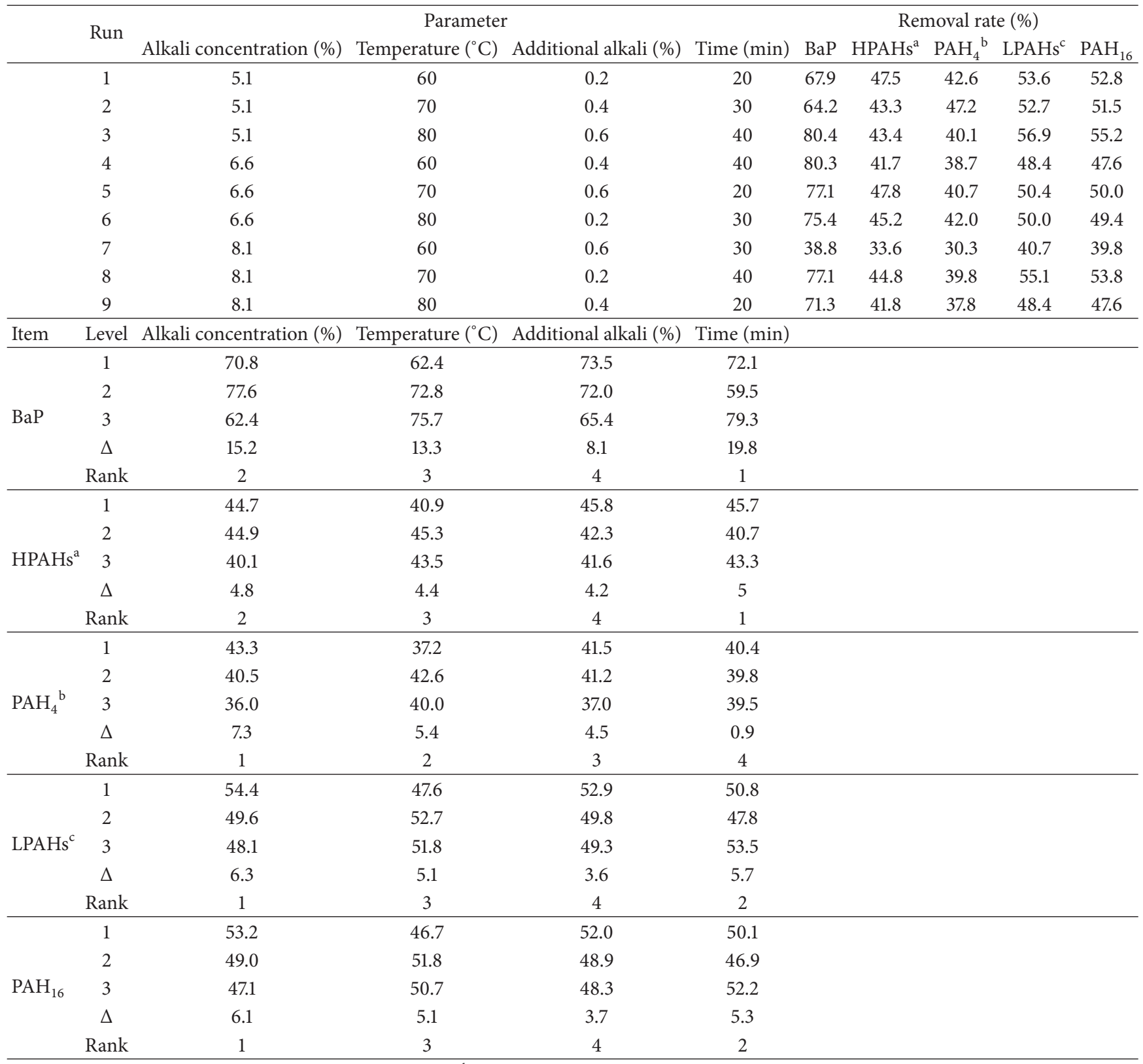

${ }^{a}$ HPAHs, including BbFlu, BkFlu, BaP, DBahA, BghiP, and IP; ${ }^{b}$ PAH4, including BaA, Chr, BbFlu, and BaP; ${ }^{c}$ LPAHs, including Nap, Acy, Ace, Fl, Phe, Ant, Flu, Pyr, BaA, and Chr.

$1.0 \%$, temperature of $120^{\circ} \mathrm{C}$, and time of $25 \mathrm{~min}$ (see Table 3). $\mathrm{S} / \mathrm{N}$ ratio analysis revealed that the optimum conditions for the five indicators were similar to the optimum conditions obtained by using the main effect plot. $\mathrm{S} / \mathrm{N}$ ratio analysis showed that WYACc amount is the highest influence parameter on all the five indicators, whereas temperature is the lowest influence parameter. Taking everything into consideration, the optimized conditions chosen were WYACc amount of $1.0 \%$, temperature of $110^{\circ} \mathrm{C}$, and time of $35 \mathrm{~min}$.

3.3. Effects of Deodorization on PAHs. Steam distillation of prepared oil sample was accomplished using the single factor experiment. temperature and time were tested; temperatures were $210,230,250$, and $270^{\circ} \mathrm{C}$ and times were $60,80,100$, and $120 \mathrm{~min}$. To study the effect of time on the deodorization efficiency, the related test was performed at different times ranging from 60 to $120 \mathrm{~min}$; similarly, to study the effect of temperature on the deodorization efficiency, the related test was performed at different temperatures ranging from 210 to $270^{\circ} \mathrm{C}$. Figures 3 and 4 show the effects of deodorization time and temperature on $\mathrm{BaP}$ and $\mathrm{PAH}_{16}$, respectively. Increase in temperature had a favourable effect on removal of $\mathrm{BaP}$ at short time of operation $(60 \mathrm{~min})$, both at 210 and $230^{\circ} \mathrm{C}$. Independently of the temperature conditions, longer time of deodorization had a more positive effect on the amount 
TABLE 3: Results of decoloration on PAHs.

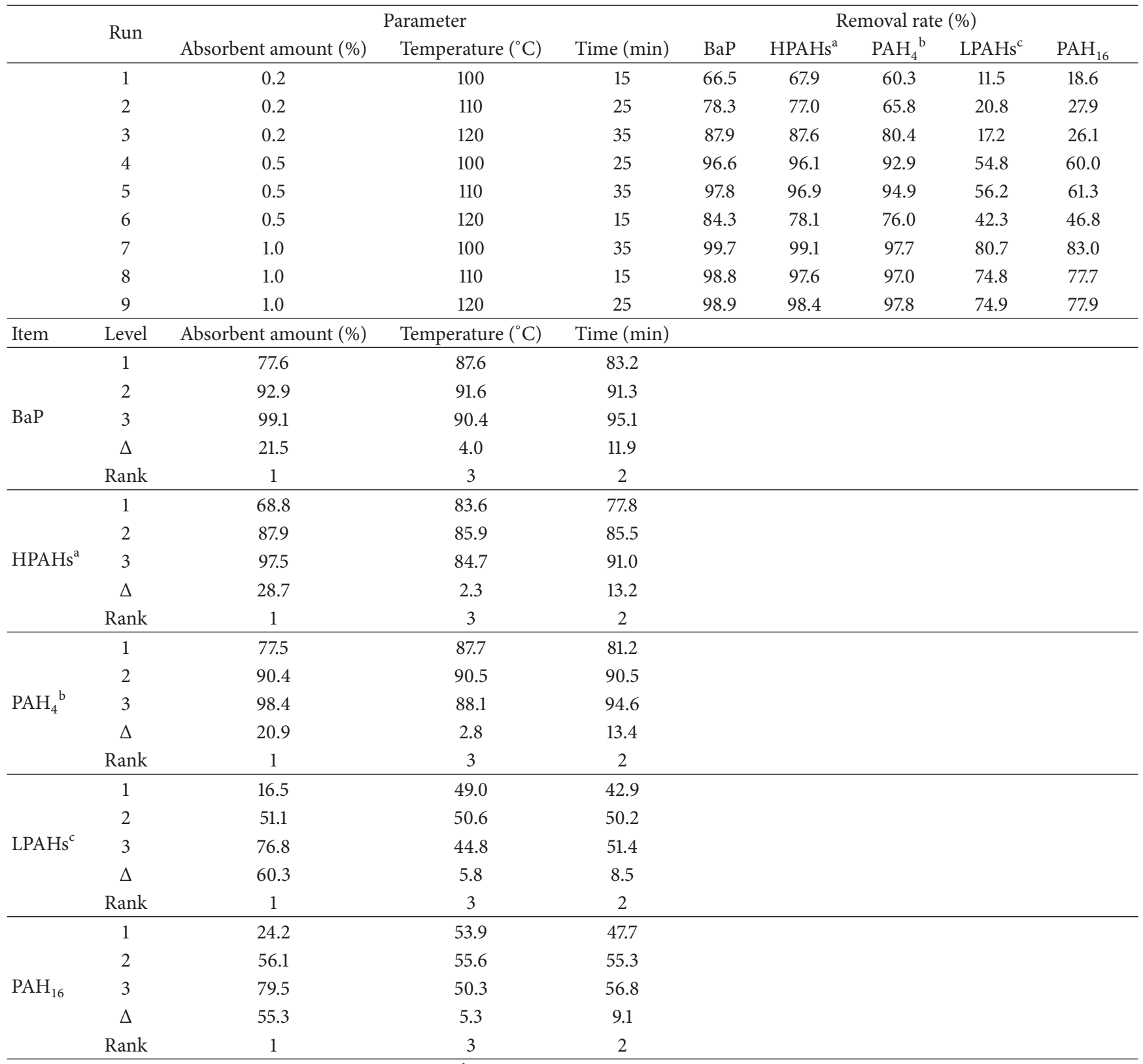

${ }^{a}$ HPAHs, including BbFlu, BkFlu, BaP, DBahA, BghiP, and IP; ${ }^{\text {PAH4}}$, including BaA, Chr, BbFlu, and BaP; ${ }^{c} \mathrm{LPAHs}$, including Nap, Acy, Ace, Fl, Phe, Ant, Flu, Pyr, BaA, and Chr.

of PAHs removed, and vice versa. The residue amounts of BaP, LPAHs, HPAHs, $\mathrm{PAH}_{4}$, and $\mathrm{PAH}_{16}$ in the deodorized oil decrease with temperature and time increase. The optimum results were achieved at temperature of $270^{\circ} \mathrm{C}$ and time of $120 \mathrm{~min}$, and the removal rates obtained were $40.8 \%, 48.4 \%$, $42.7 \%, 42.2 \%$, and $47.7 \%$.

3.4. Comparison of Effects of Three Refining Stages on PAHs. Optimum conditions obtained above from means calculation and orthogonal method must be evaluated through real experiment to check the accuracy of proposed optimum condition. Confirmation experiments (for neutralization and decoloration processes) were individually performed using the proposed optimum conditions from the orthogonal experiments. Concentrations of individual PAHs as well as LPAHs, HPAHs, $\mathrm{PAH}_{4}$, and $\mathrm{PAH}_{16}$ in crude oil and each step of the oil refining process are presented in Table 4 . All the analyses were carried out in triplicate and the experimental results obtained were expressed as means $\pm \mathrm{SD}$.

The amounts of $\mathrm{BaP}, \mathrm{PAH}_{4}$, and $\mathrm{PAH}_{16}$ in crude oil were $24.00 \pm 1.51,129.20 \pm 8.11$, and $763.94 \pm 16.97 \mu \mathrm{g} / \mathrm{kg}$, respectively. Meanwhile, LPAHs and HPAHs were $667.44 \pm$ 15.96 and $96.50 \pm 7.34 \mu \mathrm{g} / \mathrm{kg}$, respectively. Individual levels of PAHs ranged from $2.84 \pm 0.16$ to $212.74 \pm 8.66 \mu \mathrm{g} / \mathrm{kg}$, showing 
TABLE 4: PAHs content (mean \pm SD $(n=3))$ in crude oil and each step of the oil refining process.

\begin{tabular}{|c|c|c|c|c|}
\hline \multirow{2}{*}{ PAH } & \multicolumn{4}{|c|}{ Mean $\pm \operatorname{SD}(n=3)$} \\
\hline & Crude oil & Neutralized oil & Bleached oil & Deodorized oil \\
\hline Nap & $34.20 \pm 2.28$ & $10.75 \pm 0.78$ & $3.17 \pm 1.32$ & $4.25 \pm 0.63$ \\
\hline Acy & $5.20 \pm 0.45$ & $2.74 \pm 0.14$ & $1.06 \pm 0.01$ & $1.38 \pm 0.05$ \\
\hline Ace & $22.38 \pm 1.95$ & $4.80 \pm 0.52$ & $5.80 \pm 0.28$ & $3.61 \pm 0.11$ \\
\hline $\mathrm{Fl}$ & $67.85 \pm 5.35$ & $44.40 \pm 2.22$ & $11.05 \pm 1.00$ & $19.61 \pm 1.28$ \\
\hline Phe & $212.74 \pm 8.66$ & $47.73 \pm 2.39$ & $44.00 \pm 2.54$ & $134.40 \pm 3.32$ \\
\hline Ant & $41.67 \pm 2.56$ & $27.00 \pm 1.42$ & $4.62 \pm 0.05$ & $18.11 \pm 1.22$ \\
\hline Flu & $135.80 \pm 9.01$ & $64.60 \pm 4.03$ & $14.48 \pm 0.63$ & $77.88 \pm 6.00$ \\
\hline Pyr & $71.60 \pm 5.48$ & $39.60 \pm 2.58$ & $9.52 \pm 0.11$ & $40.95 \pm 2.33$ \\
\hline $\mathrm{BaA}$ & $38.80 \pm 2.22$ & $24.11 \pm 0.98$ & $0.54 \pm 0.01$ & $22.19 \pm 0.98$ \\
\hline Chr & $37.20 \pm 2.63$ & $22.60 \pm 0.82$ & $0.87 \pm 0.01$ & $22.09 \pm 1.36$ \\
\hline BbFlu & $29.20 \pm 1.98$ & $23.10 \pm 1.13$ & $0.27 \pm 0.01$ & $16.15 \pm 1.11$ \\
\hline BkFlu & $12.36 \pm 0.63$ & $4.24 \pm 0.51$ & $0.16 \pm 0.01$ & $7.10 \pm 0.58$ \\
\hline $\mathrm{BaP}$ & $24.00 \pm 1.51$ & $3.58 \pm 0.37$ & $0.06 \pm 0.01$ & $14.21 \pm 1.08$ \\
\hline DBahA & $2.84 \pm 0.16$ & $0.69 \pm 0.01$ & $0.05 \pm 0.01$ & $1.09 \pm 0.02$ \\
\hline BghiP & $14.10 \pm 1.13$ & $9.91 \pm 1.28$ & $0.12 \pm 0.01$ & $8.06 \pm 0.05$ \\
\hline IP & $14.00 \pm 1.18$ & $8.56 \pm 0.80$ & $0.03 \pm 0.00$ & $8.74 \pm 1.08$ \\
\hline $\mathrm{LPAHs}^{\mathrm{a}}$ & $667.44 \pm 15.96$ & $288.33 \pm 7.28$ & $95.11 \pm 3.33$ & $344.46 \pm 8.57$ \\
\hline $\mathrm{HPAHs}^{\mathrm{b}}$ & $96.50 \pm 7.34$ & $50.08 \pm 3.00$ & $0.69 \pm 0.02$ & $55.34 \pm 2.22$ \\
\hline $\mathrm{PAH}_{4}{ }^{\mathrm{c}}$ & $129.20 \pm 8.11$ & $73.39 \pm 4.82$ & $1.74 \pm 0.01$ & $74.64 \pm 2.54$ \\
\hline $\mathrm{PAH}_{16}$ & $763.94 \pm 16.97$ & $338.41 \pm 7.54$ & $95.80 \pm 4.51$ & $399.8 \pm 9.22$ \\
\hline
\end{tabular}

${ }^{a}$ LPAHs, including Nap, Acy, Ace, Fl, Phe, Ant, Flu, Pyr, BaA, and Chr; ${ }^{b} \mathrm{HPAHs}$, including BbFlu, BkFlu, BaP, DBahA, BghiP, and IP; ${ }^{\mathrm{c}} \mathrm{PAH} 4$, including BaA, Chr, BbFlu, and BaP.

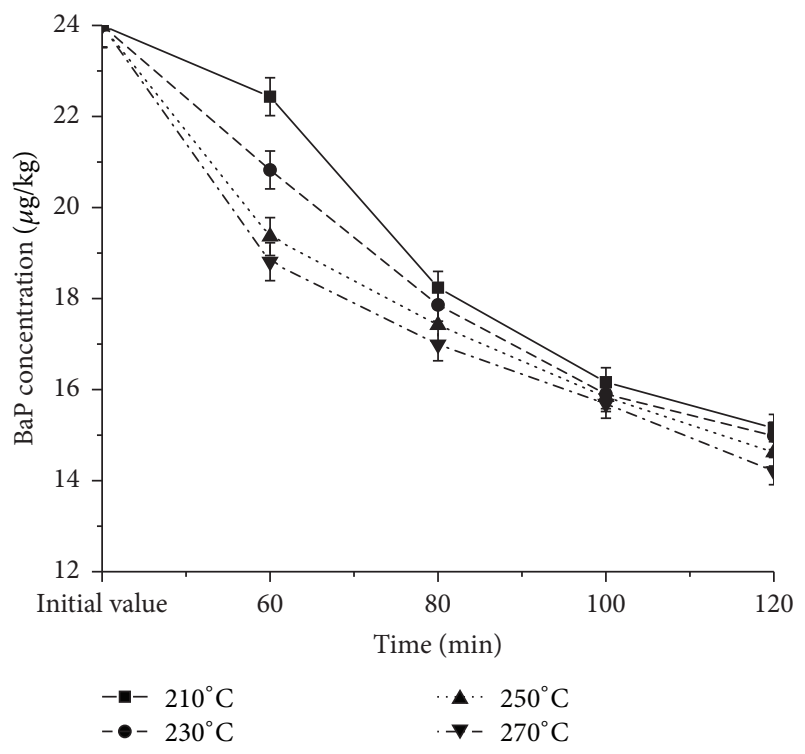

FIgURE 3: Effect of deodorization on BaP.

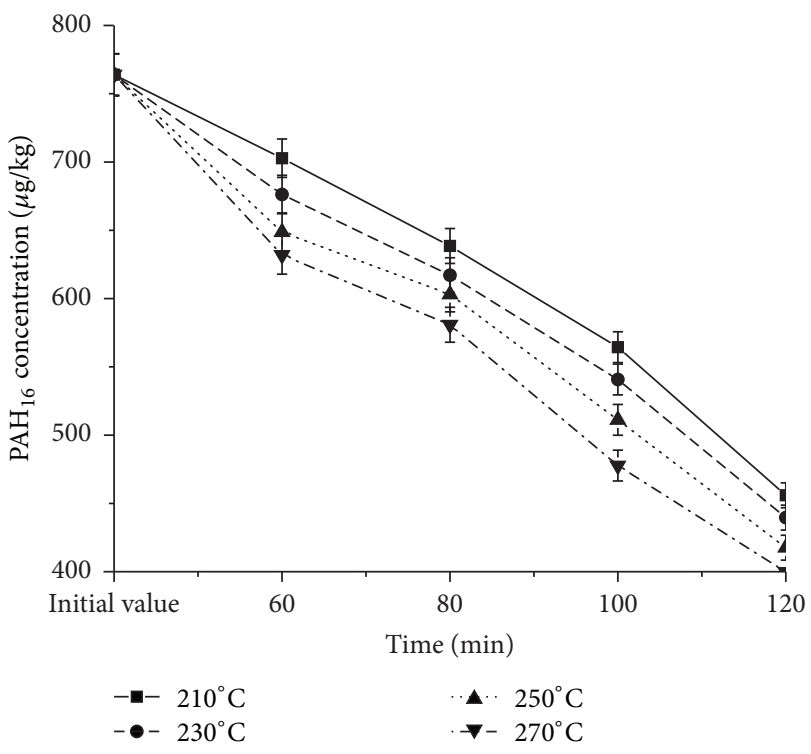

FIgURE 4: Effect of deodorization on $\mathrm{PAH}_{16}$.

Determination of other intermediary oils (neutralized, bleached, and deodorized oils) revealed that all analytes showed reduction in their levels (see Table 4). Percent reductions of intermediary oils were relative to the initial crude oil levels. As a whole, bleaching with WYACc was the most efficient means to eliminate PAHs in crude oil, while stream
Phe, Flu, and Pyr were the three kinds of compounds with highest concentrations; levels of 3 analytes were $212.74 \pm 8.66$, $135.80 \pm 9.01$, and $71.60 \pm 5.48 \mu \mathrm{g} / \mathrm{kg}$, respectively. DBahA was the lowest one oppositely, with the concentration of $2.84 \pm$ $0.16 \mu \mathrm{g} / \mathrm{kg}$. 
distillation was the worst. BaP contents decreased by $85.1 \%$, $99.7 \%$, and $40.8 \%$ in neutralized, bleached, and deodorized oils, respectively. Similarly, $\mathrm{PAH}_{4}$ contents diminished by 43.2\%, 98.7\%, and 42.2\%, respectively, and $\mathrm{PAH}_{16}$ contents decreased by $55.7 \%, 87.5 \%$, and $47.7 \%$, respectively. Previous literatures reported the decrease of PAHs during oil refining process was $27 \%-82 \%$ [11], $82 \%-84 \%$ [12], and $72 \%-87 \%$ [14], and all the oil samples (crude, neutralized, bleached, and deodorized oils) were sampled in the vegetable oil refinery. The differences among technological parameters used in the oil refining process, and the situations of samples or the laboratory conditions in refined process, may result in the small variances among the data obtained by this study and other authors.

A decrease of PAHs amounts is clearly observed from both of the LPAHs and HPAHs, in higher or lower percentage, independent of the treatments adopted. Neutralization and, chiefly, decoloration with WYACc were the more effective steps contributing to the LPAHs decrease, with the removal rates being $56.8 \%$ and $85.8 \%$, respectively. For HPAHs, decoloration was the best, with the removal rate of $99.3 \%$. Meanwhile, neutralization was also involved in the reduction of the 5-6 rings analytes, with the HPAHs contents diminished by $48.1 \%$. However, deodorization was only responsible for a slight decrease in the LPAHs and HPAHs contents, with the removal rates being $48.4 \%$ and $42.7 \%$, respectively. In comparison, other results published previously showed a more modest reduction in the HPAHs content during oil refining process: $41 \%$ [11] and 22\% [12]. Meanwhile, activated carbon was not used in any of these studies. The differences of removal rates of LPAHs during deodorization reported by this study (48.4\%) and by Cejpek et al. [11] and Larsson et al. [12] (varied between 57\% and 92\%) may be resulting from the discrepancy of operating conditions applied. Because LPAHs were in greater abundance than HPAHs in tested oil samples, the former had a higher relative contribution to the summed total PAHs. Thus, the $\mathrm{PAH}_{16}$ profile and removal behaviour observed in this study were similar to LPAHs (see Table 4).

\section{Conclusion}

Effects of neutralization, decoloration, and deodorization on PAHs during oil refining process were investigated in this study. The results of the experiment showed that the optimum conditions of neutralization were alkali concentration of $5.1 \%$, additional alkali amount of $0.2 \%$, temperature of $70^{\circ} \mathrm{C}$, and time of $40 \mathrm{~min}$. The optimum conditions of decoloration were WYACc amount of $1.0 \%$, temperature of $110^{\circ} \mathrm{C}$, and time of $35 \mathrm{~min}$ and the optimum conditions of deodorization were temperature of $270^{\circ} \mathrm{C}$ and time of $120 \mathrm{~min}$. An evident decrease of PAHs contents during oil refining process was observed, and bleaching with activated charcoal was the best to reduce PAHs in crude oil. Under the optimum experimental conditions, $\mathrm{BaP}$ contents decreased by $85.1 \%$, $99.7 \%$, and $40.8 \%$ in neutralized, bleached, and deodorized oils, respectively. $\mathrm{PAH}_{4}$ contents diminished by $43.2 \%, 98.7 \%$, and $42.2 \%$, respectively, and $\mathrm{PAH}_{16}$ contents decreased by $55.7 \%, 87.5 \%$, and $47.7 \%$, respectively. Bleaching with WYACc was the most efficient method in the comparison study to absorb PAHs in crude oil, while stream distillation was the worst. Neutralization had a modest influence on 16 analytes, with the removal rate of $55.7 \%$. However, deodorization was only responsible for a slight decrease in the LPAHs and HPAHs contents (removal rates were $48.4 \%$ and $42.7 \%$, resp.). It is worth mentioning that the high efficiency of activated charcoal indicates that the use of this material to eliminate PAHs during oil refining process is highly recommended.

\section{Conflicts of Interest}

No conflicts of interest exist in the submission of this manuscript, and the manuscript is approved by all authors for publication.

\section{Acknowledgments}

This work was supported by the Research Fund of National 13th Five-Year Plan of China (2016YFD0401405-2) and the earmarked fund for Modern Agro-industry Technology Research System (CARS15-1-10). Longkai Shi and Yulan Liu must thank Hongwei Hou from China National Tobacco Quality Supervision \& Test Center (Zhengzhou, China) for providing the LC-APPI-MS/MS system.

\section{References}

[1] S. Chumsantea, K. Aryusuk, S. Lilitchan, N. Jeyashoke, and K. Krisnangkura, "Reducing oil losses in alkali refining," Journal of the American Oil Chemists' Society, vol. 89, no. 10, pp. 1913-1919, 2012.

[2] M. V. Ruiz-Méndez, "Contribution of denaturing and deodorization processes of oils to toxic oil syndrome," ChemicoBiological Interactions, vol. 192, no. 1-2, pp. 142-144, 2011.

[3] J. Ortega-García, N. Gámez-Meza, J. A. Noriega-Rodriguez et al., "Refining of high oleic safflower oil: effect on the sterols and tocopherols content," European Food Research and Technology, vol. 223, no. 6, pp. 775-779, 2006.

[4] E. Sabah and M. Majdan, "Removal of phosphorus from vegetable oil by acid-activated sepiolite," Journal of Food Engineering, vol. 91, no. 3, pp. 423-427, 2009.

[5] R. Ajemba and O. Onukwuli, "Adsorptive removal of colour pigment from palm oil using acid activated Nteje clay. Kinetics, equilibrium and thermodynamics. Physicochem," Problem Miner. Process, vol. 49, no. 1, pp. 369-381, 2013.

[6] GB 2716-2005, "Hygienic standard for edible vegetable oil," (In Chinese).

[7] “Commission Regulation (EU) No. 835/2011 of 19 August 2011 amending Regulation EC No. 1881/2006 as regards maximum levels for foodstuffs," 2011.

[8] L.-K. Shi, Y.-L. Liu, H.-M. Liu, and M.-M. Zhang, "Onestep solvent extraction followed by liquid chromatographyatmospheric pressure photoionization tandem mass spectrometry for the determination of polycyclic aromatic hydrocarbons in edible oils," Analytical and Bioanalytical Chemistry, vol. 407, no. 13, pp. 3605-3616, 2015.

[9] L.-K. Shi, D.-D. Zhang, and Y.-L. Liu, "Incidence and survey of polycyclic aromatic hydrocarbons in edible vegetable oils in China," Food Control, vol. 62, pp. 165-170, 2016. 
[10] M. C. Rojo Camargo, P. R. Antoniolli, and E. Vicente, "Evaluation of polycyclic aromatic hydrocarbons content in different stages of soybean oils processing," Food Chemistry, vol. 135, no. 3, pp. 937-942, 2012.

[11] K. Cejpek, J. Hajslova, V. Kocourek, M. Tomaniova, and J. Cmolik, "Changes in PAH levels during production of rapeseed oil," Food Additives and Contaminants, vol. 15, no. 5, pp. 563574, 1998.

[12] B. K. Larsson, A. T. Eriksson, and M. Cervenka, "Polycyclic aromatic hydrocarbons in crude and deodorized vegetable oils," Journal of the American Oil Chemists' Society, vol. 64, no. 3, pp. 365-370, 1987.

[13] M. León-Camacho, I. Viera-Alcaide, and M. V. Ruiz-Méndez, "Elimination of polycyclic aromatic hydrocarbons by bleaching of olive pomace oil," European Journal of Lipid Science and Technology, vol. 105, no. 1, pp. 9-16, 2003.

[14] V. H. Teixeira, S. Casal, and M. B. P. P. Oliveira, "PAHs content in sunflower, soybean and virgin olive oils: evaluation in commercial samples and during refining process," Food Chemistry, vol. 104, no. 1, pp. 106-112, 2007. 

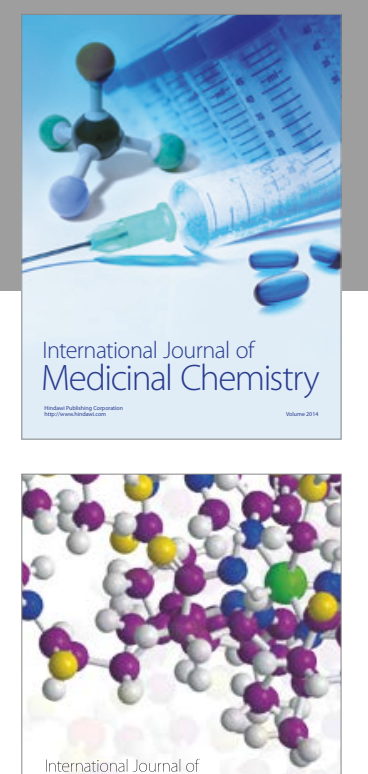

Carbohydrate Chemistry

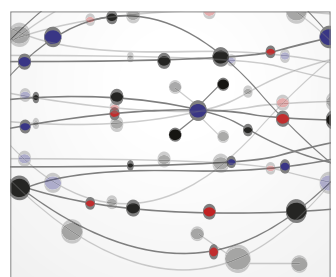

The Scientific World Journal
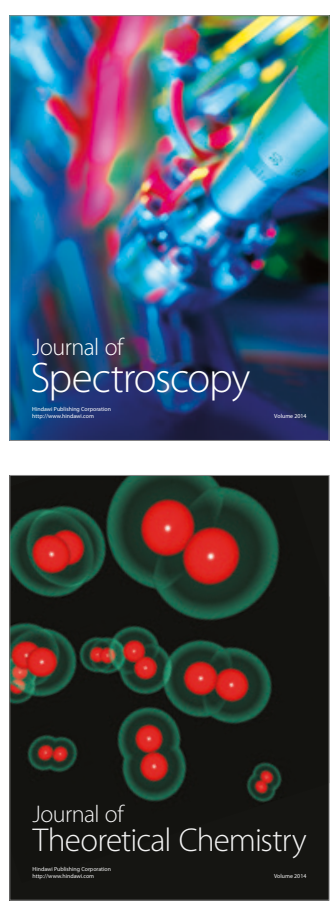
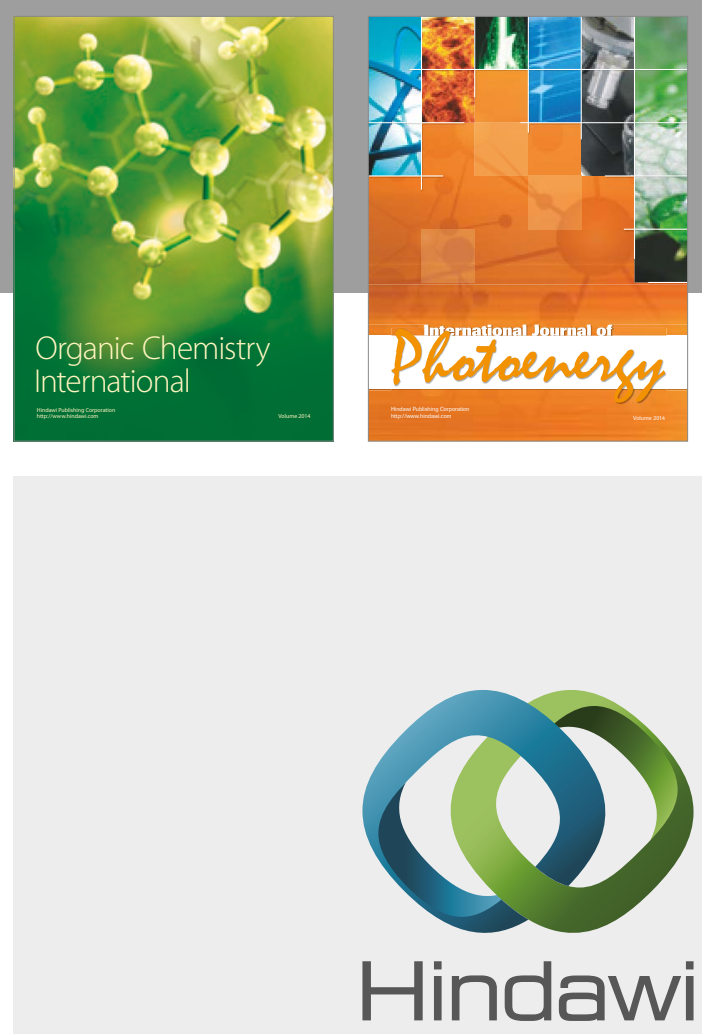

Submit your manuscripts at

https://www.hindawi.com

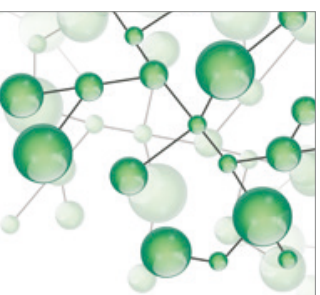

International Journal of

Inorganic Chemistry

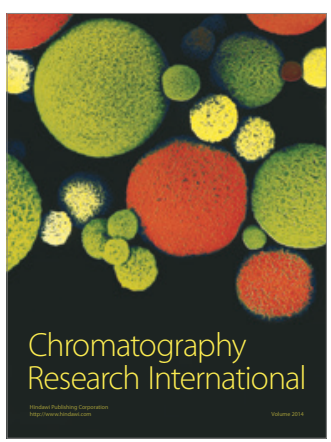

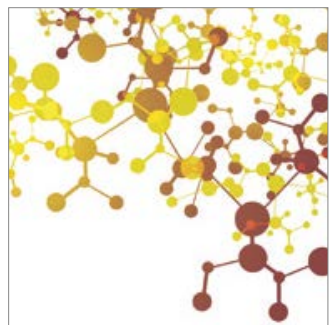

Applied Chemistry
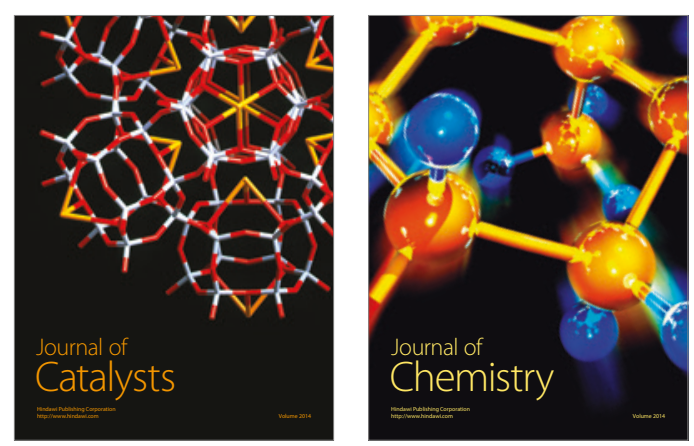
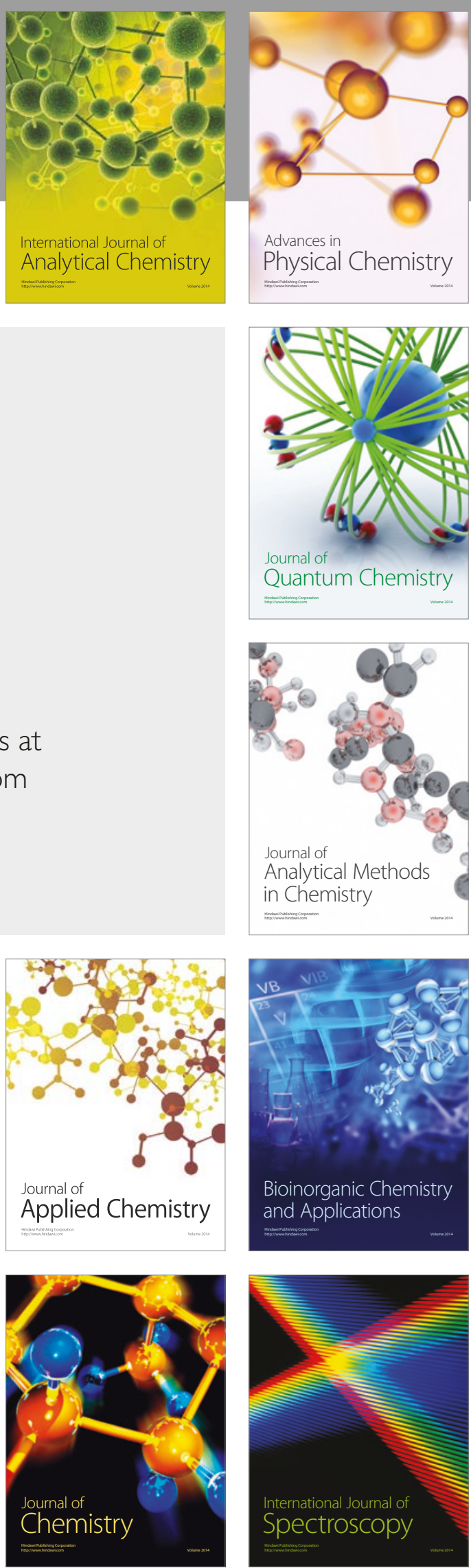